\title{
Measurements of dissipation rate in frontal zones
}

\author{
By D. CHAPMAN and K. A. BROWNING* \\ University of Reading, UK
}

(Received 21 August 2000; revised 15 May 2001)

\begin{abstract}
SUMMARY
This paper demonstrates that it is possible to use measurements of the Doppler spectrum width from a highresolution radar to obtain quantitative estimates of dissipation rate during the passage of precipitating frontal zones. Using the high-resolution Chilbolton radar, this method has been applied, for the first time, to infer the rate at which kinetic energy is dissipated by turbulence within mid- and lower-tropospheric frontal zones. Analysis of detailed measurements for one cold-frontal region showed multiple shallow sheets of high dissipation rate, with spatially averaged values around $1 \times 10^{-3} \mathrm{~m}^{2} \mathrm{~s}^{-3}$. Comparable values were also observed in a warm-frontal zone. The magnitude of the observed dissipation rate supports the hypothesis that mixing played a major role in determining the mean properties of the fronts, in that the turbulence acted to destroy the frontal shear layer on a time-scale of several hours. These issues have particular relevance to the representation of fronts within future very high-resolution numerical weather-prediction models.
\end{abstract}

KEYwordS: Dissipation rate Doppler radar Fronts Mesoscale model Mixing

\section{INTRODUCTION}

There is observational (e.g. Browning et al. 1970; Kennedy and Shapiro 1975; Blumen and Piper 1999; Chapman and Browning 1999) and theoretical (Roach 1970; Keller 1989; Blumen 1990) evidence that turbulence can balance the tendency of fronts to collapse under frontogenesis-i.e. turbulence may play a significant role in determining the properties of a frontal zone. However, some numerical weatherprediction (NWP) models do not attempt to represent the effects of turbulence in the free atmosphere in a physical manner. This is the case, for example, with the Met Office Unified Model (UM) (Cullen 1993), although it does represent boundary-induced mixing below around $2 \mathrm{~km}$. Whilst it is possible that this is acceptable with the present resolution of the model (38 levels in the vertical and a horizontal grid spacing of $12 \mathrm{~km}$ for the mesoscale version of the model), at even higher resolutions it may become necessary to represent vertical mixing explicitly at all levels in order to prevent fronts forming in the model that are unrealistically sharp.

Observations of fronts often show considerable sub-structure. It is not clear, however, whether it is this sub-structure that determines how much mixing occurs, or if the net rate of mixing is determined by the larger-scale properties of the front. Also, it is not clear whether the detailed distribution of the turbulence is important: it is conceivable that turbulence occurring in shallow sheets separated by non-turbulent layers could have different transport properties to more extensive, weaker turbulence even if the net dissipation were the same.

These issues raise the following kinds of question: are the absolute magnitudes of dissipation rate in frontal zones indeed large enough to have a major effect on the evolution of the large-scale frontal structure? Does the distribution of this turbulence need to be represented physically in predictive models and to what extent is it necessary to represent the fine-scale structures which generate the turbulence? Also, to what extent are the newer high-resolution mesoscale NWP models able to represent these finescale structures? These are the questions that motivate the present observational study. We need fine-scale observations of fronts both to understand the physical processes at

\footnotetext{
* Corresponding author: Joint Centre for Mesoscale Meteorology, Department of Meteorology, University of Reading. Whiteknights Road, PO Box 243, Reading, Berkshire RG6 6BB, UK.
}

(C) Royal Meteorological Society, 2001. 
work and to validate the increasingly high-resolution new-generation models that are becoming available. Radar can provide the necessary observations.

Whilst standard Doppler velocity measurements from radars are already known to be useful for studies of the mesoscale structure of fronts, the present study demonstrates that measurements of Doppler spectrum width can also be used to obtain turbulencerelated information on very small scales. The exceptionally high resolution of the Chilbolton microwave radar (Goddard et al. 1994) used in the present study means that it is particularly well suited to making this kind of measurement.

\section{(a) Previous measurements of mixing within fronts}

Over the last 25 years, most attempts to measure dissipation rates within frontal zones have used either aircraft or radar observations.

(i) Aircraft measurements. Aircraft measurements can provide relatively accurate point measurements of wind and thermodynamic properties at a high spatial resolution. However, there are a number of problems associated with interpreting aircraft data. Firstly, it is difficult to tell how representative point measurements are, especially in frontal regions where one expects large spatial variations. This problem is to some extent made worse by the tendency of most studies to seek out the 'interesting' regions such as those containing the strongest turbulence. Secondly, owing to the near-horizontal nature of flight tracks, vertical profiles with sufficiently high resolution are often not obtained in the regions of interest, and this makes it difficult to relate the turbulence measurements to the background atmospheric state.

The majority of reported aircraft measurements of dissipation rates in the vicinity of fronts come from upper-tropospheric regions, often around jet streams. Kennedy and Shapiro $(1975,1980)$ made a number of instrumented aircraft passes through turbulent regions within frontal-zone/jet-stream systems, measuring dissipation rates of around $20 \times 10^{-3} \mathrm{~m}^{2} \mathrm{~s}^{-3}$ (corresponding to an eddy diffusivity of around $70 \mathrm{~m}^{2} \mathrm{~s}^{-1}$ ). However, it is important to note that these are the values reported only in regions that were exceptionally turbulent, and no indication is given of how representative these measurements were of the front as a whole. Aircraft measurements by Clarke (1966) are smaller than, but not inconsistent with, these values.

(ii) Radar measurements. There are basically two methods used to derive turbulence intensity estimates from radar measurements. One of these is based on the detection of clear-air echoes from refractive-index inhomogeneities using very high frequency (VHF) radar. These echoes are caused by eddies on the scale of half the radar wavelength (a few metres) which lie within the turbulent inertial subrange. Thus, the intensity of the returned echo is related to the turbulent intensity within the radar resolution volume. However, since the resolution of VHF radars is generally much poorer than the scale of turbulent layers, the radar resolution volume will in general be only partly filled with turbulence. An accurate estimation of the dissipation rate, $\epsilon$, relies on an accurate estimation of the fraction of the volume that is turbulent, and it is not clear that this can always be obtained. Another limitation is the requirement that there be no large mean gradients in humidity, which generally limits the quantitative measurements to altitudes above a few kilometres. An example of the use of the VHF power method is given by Gage et al. (1980), who obtained a time-height distribution of $\epsilon$ during the passage of a jet stream over the radar. They found extensive regions of $\epsilon \approx 30 \times 10^{-3} \mathrm{~m}^{2} \mathrm{~s}^{-3}$ in the wind-shear regions just above and below the jet core. 
The other method of estimating $\epsilon$ using radar is to make use of the fact that turbulence within the radar pulse volume causes a spread in the spectrum of the Dopplershifted returned signal. This method is most commonly used with VHF radars for which refractive-index inhomogeneities act as tracers of the turbulent motion. However, given the relatively poor resolution of typical VHF radars (compared with the Chilbolton radar), it is doubtful that all the assumptions required of this method are generally valid. Measurements by Pepler et al. (1998) with a vertically pointing VHF radar provide time-height cross-sections through two jet-stream systems, with peak values of $2-10 \times 10^{-3} \mathrm{~m}^{2} \mathrm{~s}^{-3}$. One of the difficulties that they encountered in obtaining measurements distributed throughout the system was that the large wind speeds in the vicinity of the jet caused unacceptably large errors in the calculation of $\epsilon$ in the very regions where one would expect to find strong turbulence.

Doppler techniques can also be applied to microwave radar to estimate $\epsilon$. In this case the radar targets used to trace the air motion are usually precipitation particles. This approach was used recently by Meischner $e t$ al. (2001) to estimate $\epsilon$ within thunderstorm anvils. In an early study Bryant and Browning (1975) used microwave radar to estimate profiles of $\epsilon$ within a lower-tropospheric frontal zone using spatial spectra of the Doppler velocity. The accuracy of these measurements is, however, uncertain, as it depends on the outer scale of the turbulence spectrum being substantially larger than the maximum scale of the radar resolution volume (about $300 \mathrm{~m}$ ), a condition which their data demonstrate was not always met. Apart from this study, quantitative measurements of turbulence in lower-tropospheric frontal systems are almost non-existent. Chapman and Browning (1999) recently used the $3 \mathrm{GHz}$ microwave radar at Chilbolton to estimate the effect of large-amplitude and long-lived Kelvin-Helmholtz billows on the mean structure of a warm front. The effect was considerable, but that study used only the mean Doppler velocities and, therefore, did not exploit the full potential of the radar. The measurements that we will describe here also come from the $3 \mathrm{GHz}$ radar at Chilbolton. By exploiting its recently acquired capability to measure the Doppler spectrum width in real time, we will show that, within certain limitations, it may be used quite easily to estimate the distribution of $\epsilon$ during the passage of precipitating frontal zones. These kinds of measurement have not been made before in lower-tropospheric frontal zones.

\section{(b) Outline of this study}

In section 2 we outline and explain the method that we have used to measure $\epsilon$ using Doppler radar. In particular we show that the various assumptions required are valid in frontal regions, but probably only for radars with high spatial resolution, such as the Chilbolton radar. Section 3 describes the main case-study and we interpret the dissipation measurements in terms of the effect that the observed turbulence would have had on the structure of the front. Finally, in section 4 we discuss the implications of these results with reference to the performance of the mesoscale version of the Met Office UM. We also present the end result of another case for which we have measured $\epsilon$ during the passage of a frontal system in order to show that the results derived from the main case-study were not exceptional.

\section{Method of estimating Dissipation Rate using The ChILbOlton RadaR}

The Doppler radar data used in this study all came from the $3 \mathrm{GHz}$ multi-parameter radar (Goddard et al. 1994) which is located at Chilbolton in Hampshire $\left(51.15^{\circ} \mathrm{N}\right.$, $1.44^{\circ} \mathrm{W}$ ). The data have a range resolution of $75 \mathrm{~m}$ (at gate spacings of $300 \mathrm{~m}$ ), and a high angular resolution resulting from the $0.28^{\circ}$ two-way half-power beam width. 
The Doppler data provide information about the radial velocity of the precipitation targets. The mean Doppler velocity when the radar scans at low elevation angles is effectively the radial component of the wind field averaged over $0.25 \mathrm{~s}$, corresponding to 128 samples of the resolution volume. Previous studies using the Chilbolton radar to investigate meteorological flows (Browning et al. 1997; Chapman and Browning 1997, 1998, 1999; Chapman et al. 1998) have all used analyses of the mean Doppler velocity, but in this study we use an additional property of the field measurable with the radar.

The 128 samples obtained every $0.25 \mathrm{~s}$ allow us to calculate the Doppler spectrum width, $\sigma_{D}$. This is the standard deviation of the individual measurements used to calculate each mean Doppler velocity value, and is also equal to the power-weighted standard deviation of the radial velocities of the scatterers that lie within the resolution volume. The value of $\sigma_{D}$ is calculated and recorded in real time using the method given by Srivastava et al. (1979). This method employs the first and second autocorrelation lags to take into account the tendency for noise to increase the measured width at low signal-to-noise ratios. The Doppler spectrum width is a useful parameter to record because the variance of the radial velocity within the radar resolution volume is related to the turbulence intensity within the volume.

\section{(a) Interpretation of the Doppler spectrum width}

Turbulence is only one of a number of factors that contribute to the width of the Doppler spectrum and these other factors must be taken into account before the dissipation rate can be estimated. The factors that contribute to the measured Doppler spectrum width may be summarized as follows*:

1. Wind shear and large so-called energy-containing eddies, which create a mean gradient in Doppler velocity across the pulse volume. This can be measured by the radar from the spatial variation of the mean Doppler velocity in the vicinity of the pulse volume of interest.

2. Antenna rotation, which causes a decorrelation in the returned signals as the sampling volume changes location from pulse to pulse.

3. The drop size distribution of the precipitation targets, which will result in a range of fall speeds, a component of which is detected by an inclined radar beam.

4. Turbulence within the pulse volume.

These factors all contribute independently and so the square of the Doppler spectrum width can be expressed as the sum of the squares of all the contributing factors (including the turbulence):

$$
\sigma_{\mathrm{D}}^{2}=\sigma_{\mathrm{s}}^{2}+\sigma_{\mathrm{r}}^{2}+\sigma_{\mathrm{f}}^{2}+\sigma_{\mathrm{t}}^{2}
$$

where $\sigma_{\mathrm{s}}$ is due to shear, $\sigma_{\mathrm{r}}$ to antenna rotation, $\sigma_{\mathrm{f}}$ to differential fall speeds of the precipitation particles, and $\sigma_{\mathrm{t}}$ to turbulence (Doviak and Zrnić 1984). The individual contributions to the spectrum width are also explained in the review by Gossard (1990).

The following factors affect the relationship between the value of $\sigma_{\mathrm{t}}$ (estimated from $\left.\sigma_{\mathrm{D}}\right)$ and the value of $\epsilon$ which is derived from it:

5. Filtering of the Doppler spectrum by the beam illumination function (beam shape).

* Low signal-to-noise ratios also result in a significant broadening of the Doppler spectrum width due to contamination by ground clutter and noise. However, this effect has effectively been eliminated in this study by masking the Doppler data with the reflectivity data, so will not be considered further. 


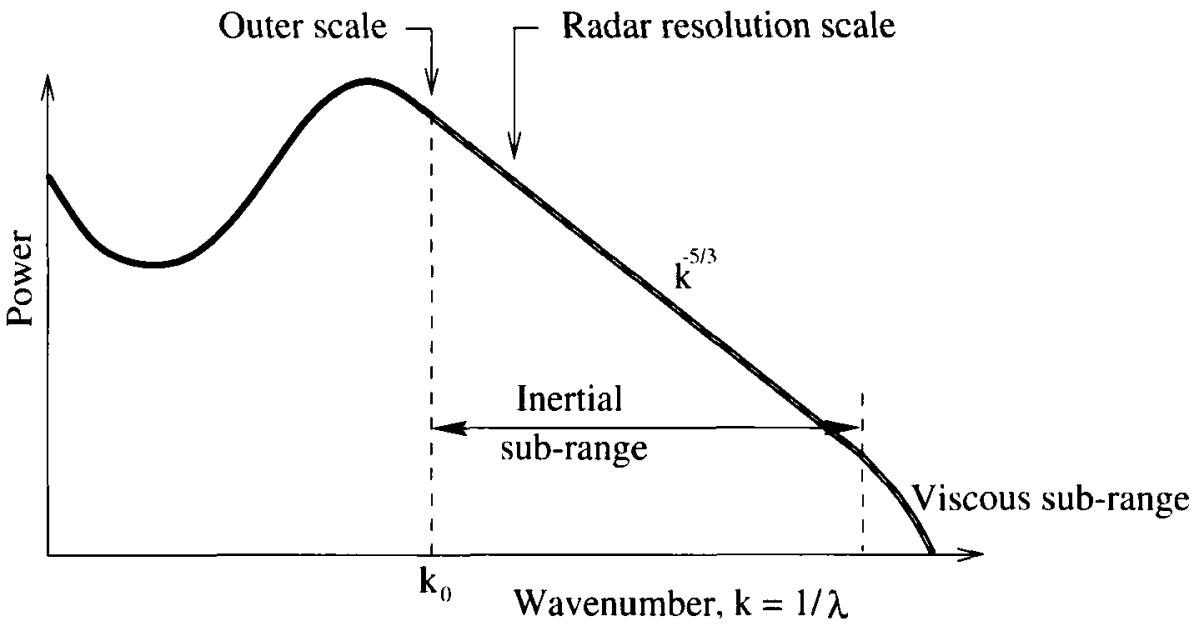

Figure 1. Schematic plot of the turbulent-energy spectrum. The overall curve indicates the kind of spectrum assumed to exist in the atmosphere. The semi-broken part of the curve, for $k>k_{0}$ (wave number), gives rise to the theoretical relationship between Doppler spectrum width, $\sigma_{\mathrm{D}}$, and dissipation rate, $\epsilon$.

6. The interaction between the turbulence outer scale length and the size of the radar resolution volume.

7. The radar detects the motion of precipitation particles, which may not always follow the air motions exactly.

These seven factors will now be considered with particular reference to the Chilbolton radar.

(i) The effect of large energy-containing eddies. Theoretical relationships between $\sigma_{\mathrm{D}}$ and $\epsilon$ (e.g. Frisch and Clifford 1974; Srivastava and Atlas 1974; Labitt 1981; Bohne 1982 ) assume that the turbulent-energy spectrum is Kolmogorov in form throughout the inertial subrange, and that no eddies exist with scales larger than the outer scale $\left(\lambda_{0}\right)$. This is the case illustrated by the semi-broken part of the curve in Fig. 1. Eddies within the inertial subrange, but with a scale larger (wave number smaller) than the maximum dimension of the radar resolution volume, will be filtered to some significant extent by the variation of the radar response across the resolution volume. This effect is taken into account in the equations presented below. However, in the real atmosphere eddies exist on scales larger than $\lambda_{0}$ (represented by the solid curve), and these may have a significant effect on $\sigma_{\mathrm{D}}$ even after they too have been filtered by the beam weighting function.

A gradient $(S)$ of radial velocity in the elevation direction of the scan results in a contribution to $\sigma_{\mathrm{D}}^{2}$ which is given by Doviak and Zrnić (1984) as

$$
\sigma_{\mathrm{s}}^{2}=\frac{\left(R \theta_{1} S\right)^{2}}{32 \log 2}
$$

where $R$ is the range and $\theta_{1}$ is the radar two-way half-power beam width $\left(0.28^{\circ}\right.$ in the case of the Chilbolton radar). At low elevation angles $S$ is effectively just the vertical shear of the radial wind, and we have calculated it at each point by performing a linear fit to the Doppler velocity data within $\lambda_{0} / 2$ above and below the data point. Outer scale lengths can be quite large in the free atmosphere; for example Brewster and Zrnić (1986) have estimated $\lambda_{0}$ as $2-3 \mathrm{~km}$, but these values are applicable only to thunderstorm 
environments. The environment in the present study was quite different and, therefore, we need to estimate $\lambda_{0}$ using Range Height Indicator (RHI) displays such as those shown later in Figs. 4 and 5 which contain layers of enhanced shear and Doppler spectrum width. The depth of these layers ranges from below the radar resolution (i.e. less than $100 \mathrm{~m}$ at a range of $20 \mathrm{~km}$ ) up to a kilometre (see also Chapman and Browning 1998). However, the majority of the layers which appear to contain most of the turbulence (i.e. those that create the most extensive regions of large $\sigma_{\mathrm{D}}$ ) tend to extend over at least $200 \mathrm{~m}$ in depth. Therefore, we estimate $\lambda_{0}$ as $200 \mathrm{~m}$, which is probably reasonable within the shear layers which cause most of the turbulence in the vicinity of the fronts we observe, even though it may not be applicable elsewhere. However, changing $\lambda_{0}$, even by a factor of two, say, has very little effect on any of the results presented in this study. Therefore, it does not appear to be a crucial parameter, at least in this context.

The component of the shear in the direction of the elevation scan (i.e. vertical for the low elevation angles used here) is almost always far greater than the component due to radial velocity shear in either the azimuthal or range directions. Therefore, we neglect the latter contributions to the Doppler spectrum width.

(ii) Antenna rotation. Doviak and Zrnić (1984) show that the variance due to antenna rotation at a rate of $\gamma$ is given by

$$
\sigma_{\mathrm{r}}^{2}=\left(\frac{\gamma \lambda \cos \phi}{\pi \theta_{1}}\right)^{2} \ln 2
$$

where $\lambda$ is the radar wavelength (approximately $10 \mathrm{~cm}$ ), and $\phi$ is the elevation angle. As the maximum rotation rate of the Chilbolton dish is only $1^{\circ} \mathrm{s}^{-1}$, the resulting correction is always less than $0.025 \mathrm{~m} \mathrm{~s}^{-1}$, which is negligible.

(iii) Differential fall speeds of the precipitation particles. When the variation in the particle fall speeds is mapped onto the Doppler velocity, the resulting contribution to $\sigma_{\mathrm{D}}^{2}$ is given by

$$
\sigma_{\mathrm{f}}^{2}=\left(\sigma_{\mathrm{f} 0} \sin \phi\right)^{2}
$$

where $\sigma_{\mathrm{f} 0}^{2}$ is the variance of the particle terminal velocities within the pulse volume. The scans analysed in this study were in rain below about $1.5 \mathrm{~km}$ and in snow above. For rain, $\sigma_{\mathrm{f} 0} \approx 1 \mathrm{~m} \mathrm{~s}^{-1}$ (e.g. Brewster and Zrnić 1986), so for the data used in this study where $\phi$ is generally less than $10^{\circ}$, the correction to the measured Doppler width is sufficiently small that we can neglect it. For snow the correction is even smaller.

(iv) Turbulence within the pulse volume. The effects (i)-(iii) described above result in the variance due to turbulence being given by

$$
\sigma_{\mathrm{t}}^{2}=\sigma_{\mathrm{D}}^{2}-\sigma_{\mathrm{s}}^{2}
$$

Factors (v)-(vii) discussed below all affect the theoretical relationships between $\sigma_{\mathrm{t}}$ and $\epsilon$. These relationships rely on the assumption that the turbulent-energy spectrum is Kolmogorov in form, and thus that turbulence is isotropic and homogeneous within the pulse volume. It is difficult to be confident that this is valid without making additional observations (for example, using aircraft measurements); however, the results given below in section 3(c) do give us some reassurance. In addition, previous comparisons between aircraft and dissipation rates derived from Doppler spectrum-width data have shown excellent agreement (e.g. Bohne 1981), even when using data from radars with much poorer resolution than the Chilbolton radar. 
(v) Beam filtering. The radar has a Gaussian-type distribution of sensitivity within the resolution volume, and this results in a filtering of the turbulence spectrum (especially on scales comparable to or larger than the resolution volume), and thus a potentially significant effect on the relationship between $\epsilon$ and the measured $\sigma_{\mathrm{D}}$. This is described by Doviak and Zrnić (1984), and the effect is included in Eq. (6) below.

(vi) The relationship between the outer scale, $\lambda_{0}$, and the size of the radar resolution volume. Bohne (1982) assumed that the effects of eddies on scales larger than $\lambda_{0}$ had been removed from the data (as described in (i) above). Then, if $\lambda_{0}$ is smaller than the maximum dimension of the resolution volume, $\lambda_{0}$ needs to be known if $\epsilon$ is not to be substantially underestimated. If we assume that $\lambda_{0}$ is around $200 \mathrm{~m}$, then so long as we use data only at ranges less than $30 \mathrm{~km}$, the maximum size of the resolution volume will be smaller than $\lambda_{0}$. Thus we do not have to make a correction involving the value of $\lambda_{0}$ (other than as described in (i) above). This is important because, if we did have to make such a correction, Bohne showed that an error of a factor of, say, two in our estimate of $\lambda_{0}$ could lead to an even larger error in the calculation of $\epsilon$.

(vii) Imperfect precipitation-particle response. In convective situations the radar echo is often dominated by large rain drops or hail particles because of the dependence on $D^{6}$, where $D$ is the particle diameter. Such particles respond mainly to the larger eddy scales. At close ranges, where the radar resolution volume is relatively small, it is therefore possible that eddies on scales smaller than the resolution volume will not be well represented by such precipitation particles. Quantitative estimation by Bohne (1982) of the magnitude of this effect suggests that it will result in an underestimation of $\epsilon$ by up to around $30 \%$ in regions of substantial turbulence occurring within heavy rain at short range. However, in the data we use here, the precipitation is generally light rain or snow, so the underestimation of $\epsilon$ due to the imperfect precipitation response is unlikely to be large (perhaps on average up to around $10 \%$ ). We, therefore, neglect this correction in our analysis.

In summary, we need to take into account the background wind shear to derive $\sigma_{\mathrm{t}}$ from the measured Doppler spectrum width (Eq. (5)), but when we calculate $\epsilon$ from $\sigma_{\mathrm{t}}$ we are justified in neglecting the effects of imperfect precipitation response and the finite turbulence outer length scale. Thus, we can use the analytically derived expressions of Frisch and Clifford (1974) (after making a correction as given by Labitt (1981)):

$$
\sigma_{\mathrm{t}}^{2}=1.345 C(\epsilon \alpha)^{2 / 3}\left(1-\frac{1}{15} z-\frac{1}{105} z^{2}-\frac{5}{1701} z^{3} \ldots\right)
$$

for longer ranges where $\beta<\alpha$ and $z=\left(1-\beta^{2} / \alpha^{2}\right)$, or

$$
\sigma_{\mathrm{t}}^{2}=1.345 C(\epsilon \beta)^{2 / 3}\left(1-\frac{4}{15} z-\frac{8}{105} z^{2}-\frac{64}{1701} z^{3} \ldots\right)
$$

for short ranges where $\alpha<\beta$, and $z=\left(1-\alpha^{2} / \beta^{2}\right)$. The beamwidth $(\alpha)$ and pulse length $(\beta)$ parameters are given by

$$
\begin{aligned}
& \alpha=\frac{R \theta_{1}}{(8 \log 4)^{1 / 2}} \\
& \beta=\frac{L}{(8 \log 4)^{1 / 2}}
\end{aligned}
$$

where $R, \theta_{1}$ and $L$ are the radar range, two-way half-power beam width and half-power pulse length, respectively. According to Bohne (1982), these relationships should hold 


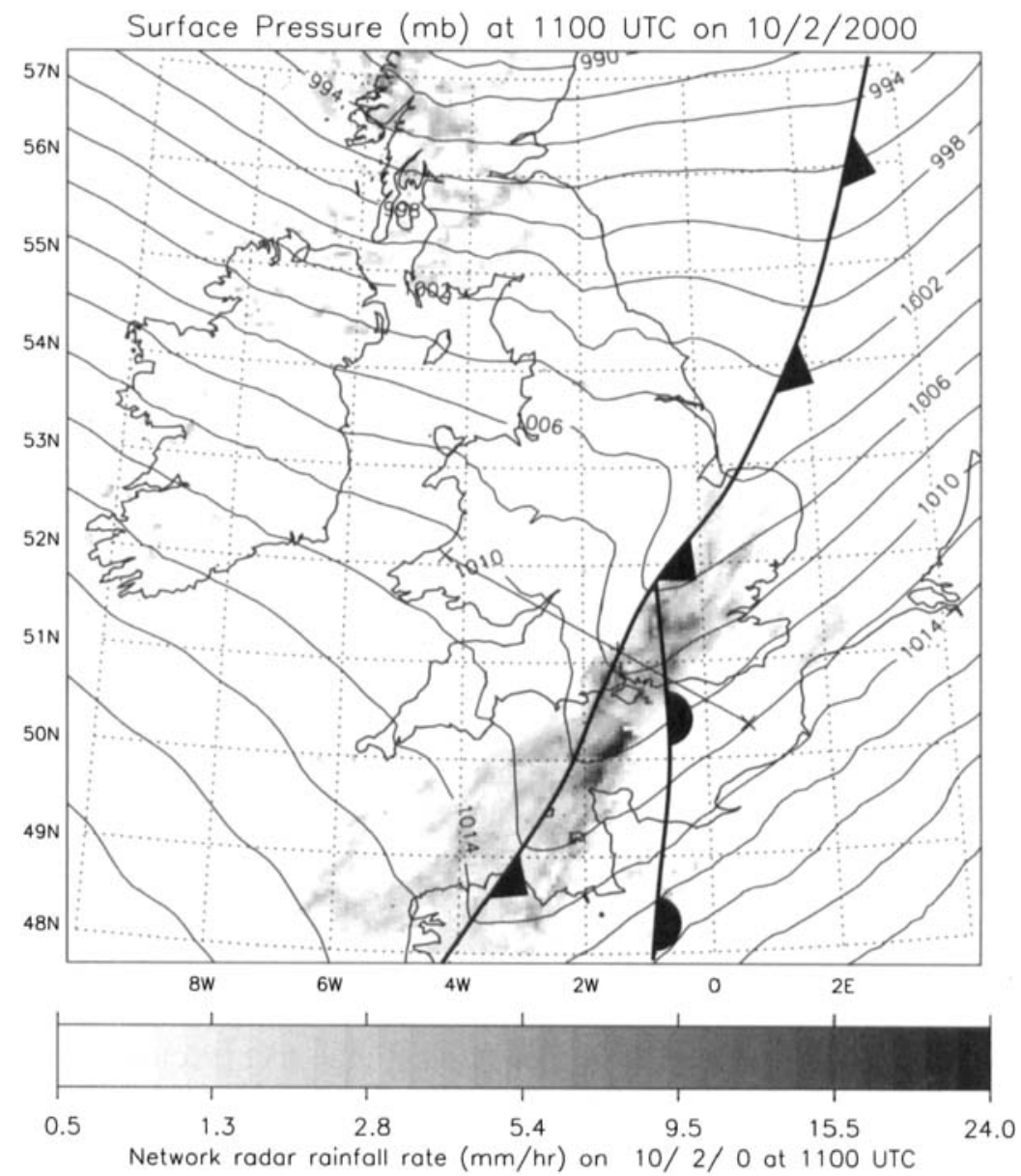

Figure 2. UK radar-network rainfall rate and Unified Model mean-sea-level pressure and location of the surface fronts at 1100 UTC on 10 February 2000. The line XX at right angles to the cold front indicates the location of the model cross-section shown later in Fig. 11. The + symbol where the line XX intersects the surface cold front indicates the location of the Chilbolton radar.

reasonably well at ranges where the maximum size of the resolution volume is smaller than the outer scale of the turbulence. Assuming $\lambda_{0} \geqslant 200 \mathrm{~m}$, this means that we can confidently use the expressions in Eqs. (6) and (7) to calculate $\epsilon$ out to a maximum range of $30 \mathrm{~km}$, without having to know the exact outer scale length.

\section{THE MAIN CASE-STUDY}

\section{(a) General situation and radar scanning strategy}

On 10 February 2000 a strong cold anafront passed over the UK producing a broad area of light rain with embedded regions of heavier rain due to narrow cold-frontal rain bands, each of which was associated with weak line convection at the surface. This general rain area is illustrated in Fig. 2, which shows the rainfall rate from the UK weather radar network at 1100 UTC, together with the NWP model-derived analysis of mean-sea-level pressure and the location of the main cold front. The cold front was travelling at a speed of $16 \mathrm{~m} \mathrm{~s}^{-1}$ (approximately $1 \mathrm{~km} \mathrm{~min}^{-1}$ ) towards the south-east at this time. A more detailed analysis indicates that the surface cold front in the model 
(and thus shown in Fig. 2) lagged behind the observed location of the cold front by just under one hour; this should be borne in mind later when we compare radar and model data.

The Chilbolton radar data that we use consist of a systematic sequence of RHIs (equivalent to vertical cross-sections) and Plan Position Indicators (PPIs, where the radar scanned in azimuth at a constant elevation angle). The RHIs were obtained at azimuth intervals of about $18^{\circ}$ between 0 and $360^{\circ}$ from north. The PPIs were obtained at an elevation angle of $1^{\circ}$. It took 28 minutes to complete each cycle of 20 RHIs and one PPI, during which time the front travelled about $27 \mathrm{~km}$.

The first radar cross-section that we present (Fig. 3) passed through the surface cold front almost perpendicularly (along part of the line XX in Fig. 2) at 1111 UTC just after the surface cold front had passed over the radar. The Doppler velocity (in this case effectively the horizontal component of the radial wind velocity) has been shaded so that the darkest blue indicates air moving to the south-east (towards the right in this section), and red indicates air moving towards the north-west relative to the advancing frontal system. The surface cold front (SCF) was accompanied by two surface convergence lines; one of these is seen in Fig. 3 to have been located at a range of about $53 \mathrm{~km}$ from the radar between a layer of cold air moving towards the south-east and warm boundarylayer air, with a relative velocity towards the north-west, the latter being at least partly below the radar horizon at ranges greater than $58 \mathrm{~km}$. Evidence from RHIs obtained when this convergence line was close to the radar (not shown), suggests that this warm air ascended suddenly up to around $800 \mathrm{~m}$, and then, after a brief period of descent, continued to ascend more gently whilst moving relatively to the north-west above the cold air. Descent and ascent are inferred from the direction of the relative flow in relation to the inclined layers of flow; although this is a crude approach it nevertheless gives a flow pattern that is consistent with other observed cases and with the conceptual model shown in Fig. 8.8 of Browning (1990). Figure 3 also shows evidence of a number of other shallow circulations in this cross-frontal plane. Other RHIs show that at least two of these circulations connected to the ground and were associated with line-convection elements. The detailed nature of these circulations is largely incidental to this study, so will be described elsewhere.

\section{(b) Dissipation rate estimates}

Our goal is to obtain spatially representative profiles of $\epsilon$ by averaging a very large number of estimates obtained from RHIs in all 20 directions from the radar over a horizontal area comparable with the resolution of the mesoscale model. However, before we do this it is informative to look at the distribution of $\epsilon$ within individual RHIs. We will show two more RHIs, one perpendicular (Fig. 4) and one parallel (Fig. 5) to the surface cold front, each of which is at least qualitatively representative of other similarly orientated cross-sections. The locations of these cross-sections are shown in Fig. 6 , relative to the position of one of the surface line-convection segments where there is a cross-frontal circulation resembling that in the cold-front model of Browning (1990).

The cross-section towards the north-west in Fig. 4 corresponds to A1-A2 in Fig. 6, and extends the previous cross-section (Fig. 3, orientated towards the south-east) further to the north-west, behind the surface front. The shading of the Doppler velocity (Fig. 4(a)) has again been chosen so that blue represents air travelling rapidly from left to right towards the SCF (located $30 \mathrm{~km}$ to the south-east at this time), and orange/red represents air travelling rearwards away from the SCF. The multiple layers noted earlier can be seen clearly in this section, too. 


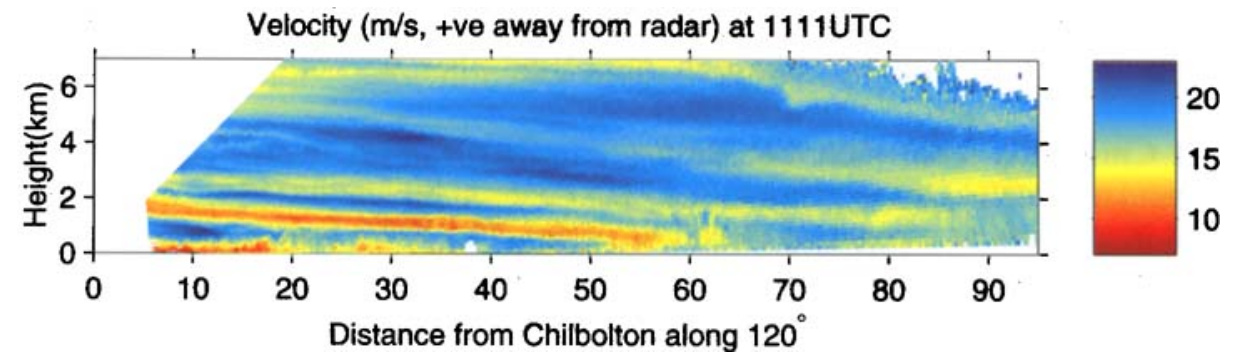

Figure 3. Radar cross-section (Range Height Indicator) through the surface cold front at 1111 UTC. The shading shows Doppler velocity ( $\mathrm{m} \mathrm{s}^{-1}$, positive away from the radar).

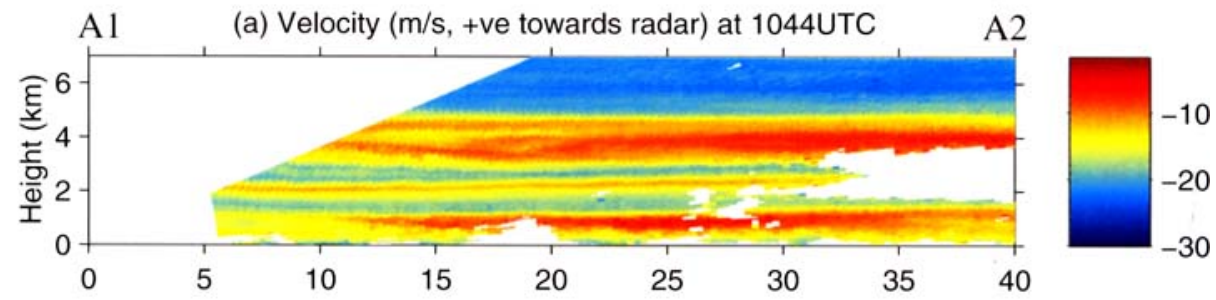

(b) $\log _{10}(\varepsilon)$ at 1044 UTC

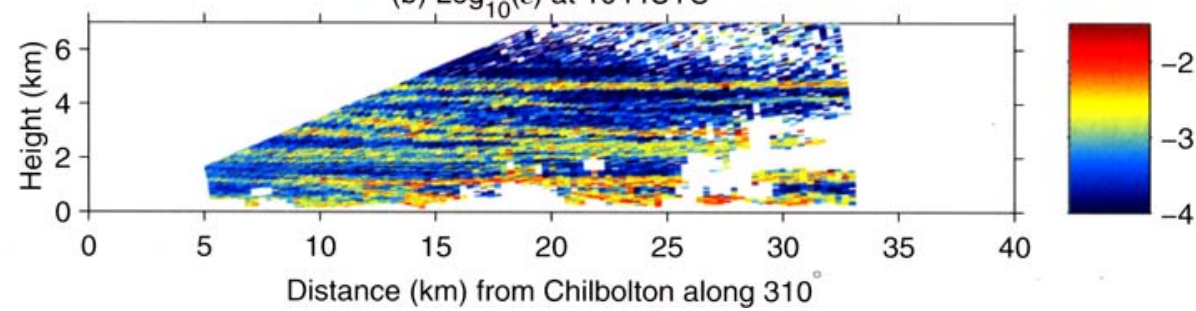

Figure 4. Radar cross-section (Range Height Indicator) orientated perpendicular to and looking away from the surface cold front at 1044 UTC. (a) Doppler velocity $\left(\mathrm{m} \mathrm{s}^{-1}\right.$, positive toward radar). (b) The logarithm of the dissipation rate, $\epsilon$, (where $\epsilon$ is in $\mathrm{m}^{2} \mathrm{~s}^{-3}$ ) calculated using $\mathrm{Eq}$. (6).

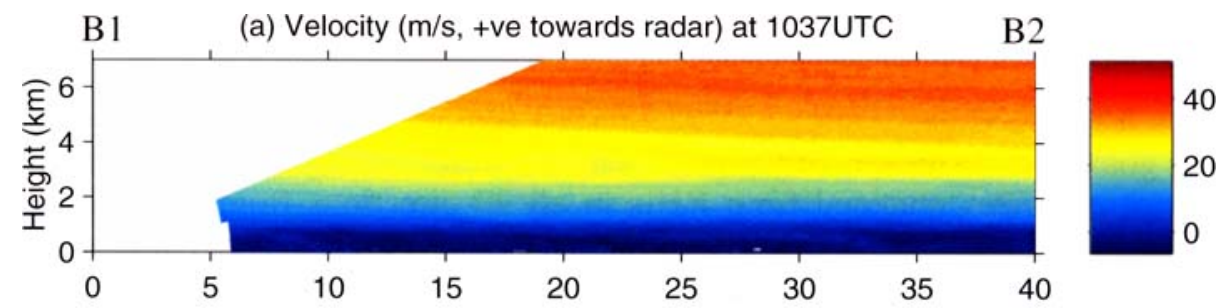

(b) $\log _{10}(\varepsilon)$ at 1037 UTC

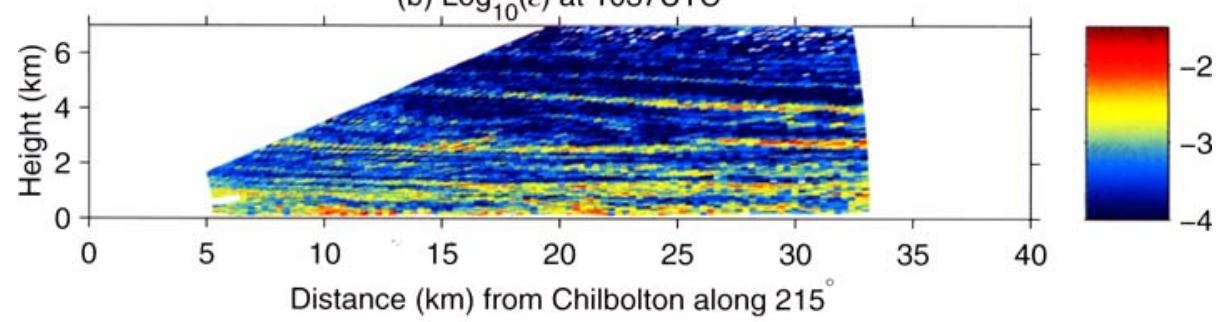

Figure 5. Radar cross-section (Range Height Indicator) orientated almost parallel to the surface cold front at 1037 UTC. (a) Doppler velocity ( $\mathrm{m} \mathrm{s}^{-1}$, positive toward radar). (b) The logarithm of the dissipation rate, $\epsilon$, (where $\epsilon$ is in $\mathrm{m}^{2} \mathrm{~s}^{-3}$ ) calculated using Eq. (6). 


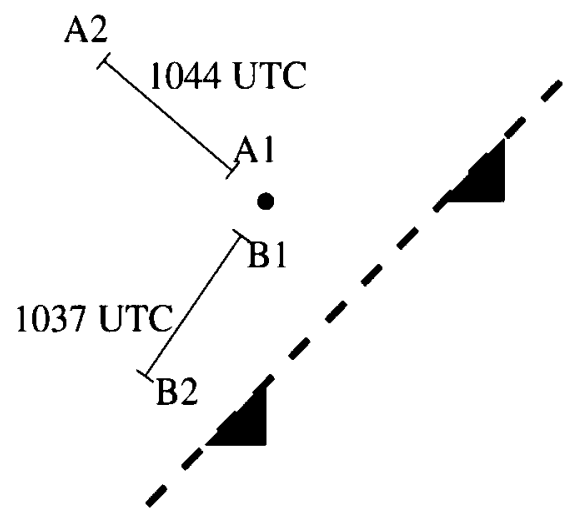

Figure 6. Locations and times of the radar cross-sections in Figs. 4 and 5, relative to the surface location of one of the line-convection segments having a cold-frontal flow structure.

Figure 4(b) shows the dissipation rate, $\epsilon$, which is considered accurate out to a range of only $30 \mathrm{~km}$ for reasons given in section 2(a). This shows that the turbulence in the vicinity of the frontal zone is confined almost exclusively to very shallow sub-layers (sheets) which are separated from each other by regions of much reduced turbulence (the dissipation rate within each sub-layer can be more than an order of magnitude greater than in the regions between them). The data-free (white) regions, especially beyond $25 \mathrm{~km}$, occur where there was very little signal (Doppler spectrum widths have been masked where the reflectivity was less than $0 \mathrm{dBz}$ ).

Figure 5 shows the Doppler velocity and $\epsilon$ for the cross-section B1-B2 almost parallel to the front. In this direction the Doppler velocity pattern is much less layered, with the main feature being a broad shear layer between the surface and around $6 \mathrm{~km}$. This is consistent with the expected thermal wind shear across such a front, although on closer inspection this broad shear layer contains many sub-layers where the shear is more concentrated. The dissipation rate (Fig. 5(b)) again consists of distinct sub-layers of very much enhanced turbulence which are continuous with the sub-layers shown in the perpendicular section in Fig. 4.

Since we are interested in the effect of the turbulence on the frontal structure on scales comparable with the resolution of the mesoscale model, we have averaged the data from each cycle of 20 vertical cross-sections (RHIs) to give a single vertical profile of $\epsilon$, representing the dissipation rate averaged over a volume extending between 5 and $30 \mathrm{~km}$ from the radar. These profiles have been obtained by masking the individual $\epsilon$ cross-sections with the radar reflectivity (to remove the misleadingly high Doppler spectrum widths in regions of low signal), and then averaging the data into $100 \mathrm{~m}$ deep bins distributed in the vertical. Before averaging, a small range-dependent height correction (section 3(c)) was applied so as to be able to resolve the individual sloping sub-layers within the average profiles. A sequence of eight such profiles of $\epsilon$, obtained every 28 minutes during the passage of the cold front, are displayed as a time-height cross-section in Fig. 7(a). Each of these profiles is based on a total of at least 4000 values of $\epsilon$ in each height bin (apart from above around $3 \mathrm{~km}$ before 0930 UTC), and so the resulting profiles are regarded as being very representative of the frontal region as it passed close to the radar. If one assumes that the front was in a steady state during its passage over the radar, this time-height cross-section can also be viewed as if it were a conventional spatial cross-section from north-west (left) to south-east (right) through the front. 
(a) $\log _{10}(\varepsilon)$

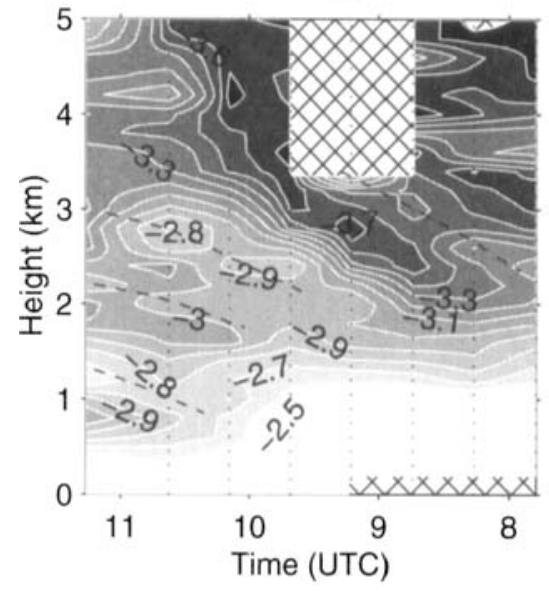

(b) Vertical wind shear $\left(\mathrm{m} \mathrm{s}^{-1} \mathrm{~km}^{-1}\right)$

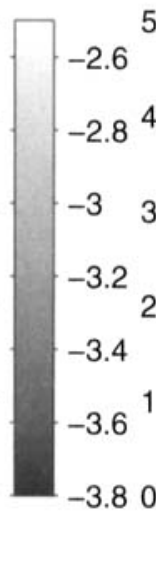

(b) Vertical wind shear ( $\mathrm{m} \mathrm{s}^{-1} \mathrm{~km}^{-1}$ )

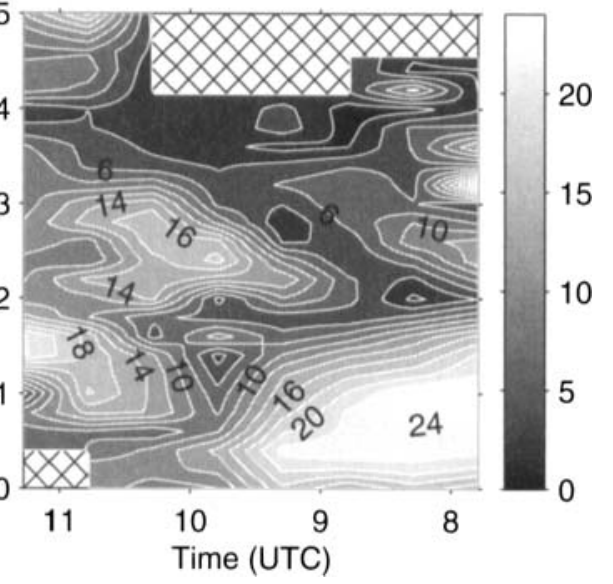

Figure 7. Time-height sections of (a) the logarithm of the dissipation rate, $\epsilon\left(\mathrm{m}^{2} \mathrm{~s}^{-3}\right)$ and (b) vertical wind shear $\left(\mathrm{m} \mathrm{s}^{-1} \mathrm{~km}^{-1}\right)$ during the passage of the cold front. Vertical dotted lines in (a) show the times of six of the eight profiles; there are also profiles at 0748 and 1115 UTC (on right and left edges of figure). The hatched regions show where there were insufficient data. The dashed lines indicate the sub-layers or sheets of enhanced dissipation rate.

Figure 7(b) shows the corresponding time-height section of the distribution of vertical wind shear within the front. This has been calculated by fitting the mean Doppler data to a sinusoid (after making height corrections and reorganization into bins as described above), along the lines of the Velocity Azimuth Display (VAD) method suggested by Lhermitte and Atlas (1961). This then gives the wind velocity averaged over a radius of $30 \mathrm{~km}$ from the radar, every 28 minutes, from which the vertical wind shear can easily be derived. The relationship between the profiles of wind shear and $\epsilon$ is discussed in section 3(d).

\section{(c) Validation}

Before going on to interpret the dissipation rate measurements in terms of the meteorological impact of the turbulence within the frontal region, we now reconsider the validity of some of the main assumptions which have been made in the derivation of $\epsilon$ from $\sigma_{\mathrm{D}}$.

(i) Range dependence of $\epsilon$. All of the possible sources of contamination of the Doppler spectrum width make contributions that are dependent on the range, so if any of these factors has been either incorrectly accounted for or unjustifiably neglected, one might expect to see a systematic range effect in the calculated values of $\epsilon$. Therefore, we have calculated a range profile of $\epsilon$ using all 160 RHIs over the period used in the derivation of Fig. 7, and the result is shown in Fig. 8. The flatness of this curve gives us some confidence that we are accounting correctly for at least the important factors affecting the Doppler spectrum width.

(ii) Isotropy and wind-shear correction. We can see that within individual sheets of turbulence in the main frontal zone (Figs. 4(b) and 5(b)), the estimate of $\epsilon$ shows no appreciable variation with range either in the cross- or along-front directions. Therefore, if we assume that the regions sampled by section A1-A2 and B1-B2 have identical turbulent properties, then we effectively have measurements of $\epsilon$ obtained in orthogonal 


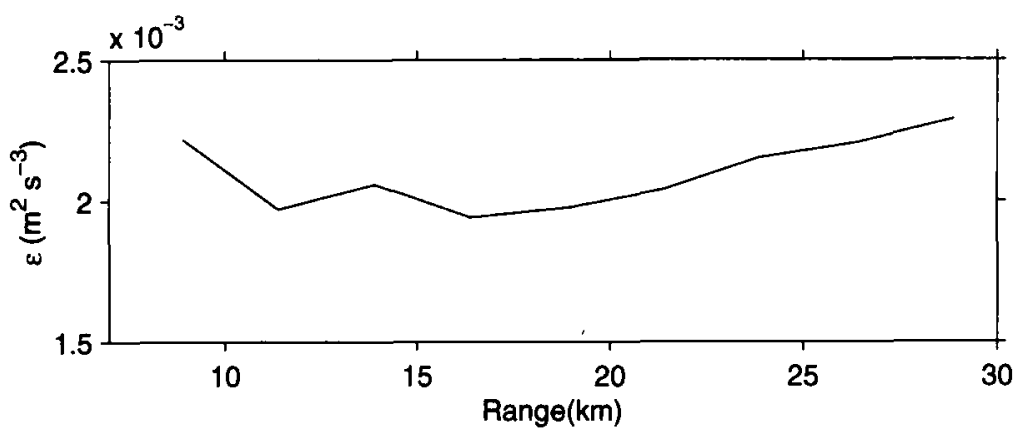

Figure 8. Range dependence of dissipation rate $(\epsilon)$, calculated by averaging over all radar estimates at all heights below $3.2 \mathrm{~km}$ and between ranges 9 and $30 \mathrm{~km}$.

directions through what can be considered as a single region of turbulence. We wish to check that the turbulence on scales that contribute to the Doppler spectrum width in this region is isotropic.

In order to simplify the comparison of Figs. 4(b) and 5(b), we have reduced each cross-section to a profile of $\epsilon$. Because the sheets of enhanced turbulence are sloped, a simple horizontal averaging of the data in these cross-sections would have blurred the distinction of individual sheets. As we wish to preserve the structure associated with individual sheets (which are particularly apparent in the front-perpendicular section), the following height adjustment was made before averaging:

$$
\Delta z=R \alpha \cos \left(\beta-135^{\circ}\right)
$$

where $\beta$ is the azimuthal direction of the cross-section, $R$ is the range (in $\mathrm{km}$ ) of each data point, and $\Delta z$ is the height adjustment which must be made to each data point. The slope of the layers (observed to vary approximately linearly with height) is given by

$$
\alpha= \begin{cases}0.007 z & \text { if } 0.007 z<0.02 \\ 0.02 & \text { otherwise }\end{cases}
$$

where $z$ is the original altitude (in $\mathrm{km}$ ) of each data point. After making this adjustment, the profiles were obtained by averaging horizontally within each cross-section. Figure 9(a) shows the resulting profiles of $\epsilon$, the solid and dashed lines indicating the profiles obtained from front-parallel and front-perpendicular cross-sections, respectively. There is clearly a very good correlation between the two profiles of $\epsilon$ at all heights up to $5 \mathrm{~km}$, especially in regard to the altitudes of the peaks and troughs. Apart from a small offset (which could be due to a difference in turbulence magnitude between the locations of the two sections), the estimated profile of $\epsilon$ is nearly independent of azimuth. There are two possible reasons why there could be such a good correlation:

1. The turbulence is essentially isotropic on scales smaller than the resolution volume, so our estimate of $\epsilon$ is independent of the viewing azimuth.

2. The individual peaks and troughs in the two profiles could be caused by inadequate correction for the effects of another property of the flow, notably the vertical wind shear. We have already noted that the wind shear can in some circumstances make a significant contribution to the $\sigma_{\mathrm{D}}$; perhaps this contribution is not being accounted for correctly.

We have already shown some evidence in section 3(c)(i) that argues against contamination by vertical wind shear. To assess further the likelihood of such contamination, we 
(a)

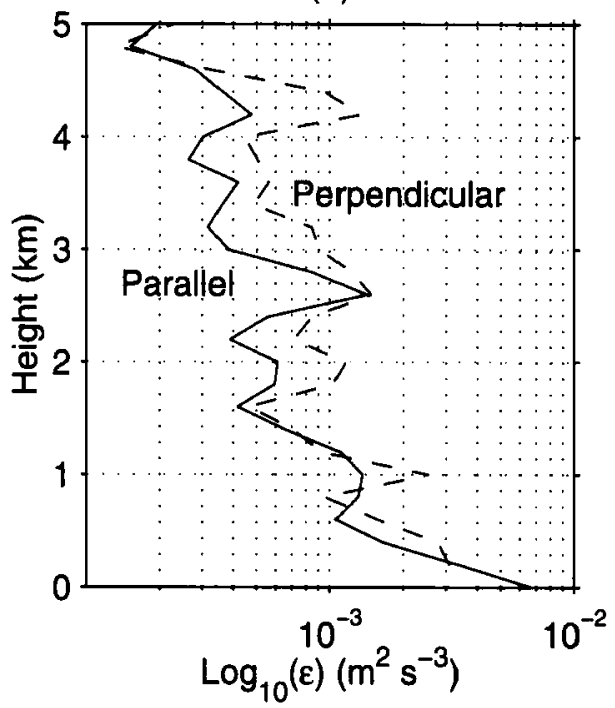

(b)

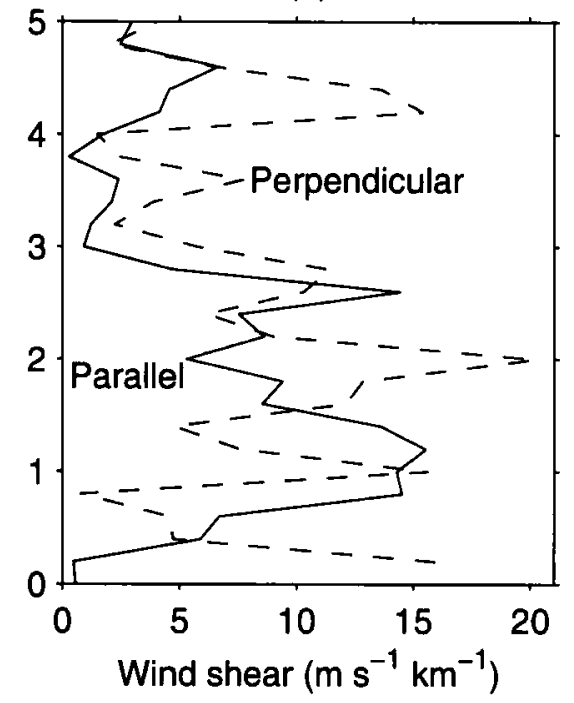

Figure 9. Vertical profiles of (a) the logarithm of the dissipation rate ( $\epsilon$ ) and (b) vertical shear of the radial component of the wind derived from cross-sections oriented parallel (solid lines) and perpendicular (dashed lines) to the front.

have derived Fig. 9(b) which shows the distribution of the magnitude of the vertical shear of the front-parallel and perpendicular wind components (after making the same height correction as in Fig. 9(a)). In comparing Figs. 9(a) and (b), we note that between 2.5 and $5 \mathrm{~km}$ there is a correlation between the peaks and the troughs of all four $\epsilon$ and shear profiles. This is exactly what one might expect for shear layers which are not exactly orientated either parallel or perpendicular to the front, as there will be a component of shear in both directions, and one might expect to find the enhanced turbulence within layers of enhanced wind shear. In contrast, between heights 1.0 and $2.5 \mathrm{~km}$ we see that the peaks and troughs in the two wind-shear profiles (Fig. 9(b)) are, if anything, anti-correlated. For example, the layer of enhanced front-perpendicular shear at $2 \mathrm{~km}$ coincides with a minimum in the front-parallel shear. In spite of this, the two profiles of $\epsilon$ in Fig. 9(a) remain extremely well correlated with one another around this height. In particular, the front-parallel component of $\epsilon$ at $2 \mathrm{~km}$ is not disproportionately smaller than the corresponding front-perpendicular component of $\epsilon$, despite the front-parallel component of the shear being almost four times smaller than the corresponding frontperpendicular component of the shear. This indicates that:

- the contribution of the vertical shear of the radial component of the wind is being removed correctly from the Doppler spectrum width measurement. This implies that

- the shallow sub-layers (sheets) of enhanced $\epsilon$ that we see are due to properties of the wind field on scales smaller than the resolution volume, and that

- the turbulence detected by the radar is, to a first approximation, isotropic on scales smaller than the resolution volume.

These findings have been reproduced using many other radar cross-sections. Taken together with the range invariance of $\epsilon$ shown earlier in Fig. 8, this gives us confidence that the main assumptions involved in the derivation of $\epsilon$ from $\sigma_{\mathrm{D}}$ are in fact justified. 


\section{(d) Interpretation of the results in terms of the effect of dissipation on the frontal} structure

Having gained confidence in section 3(c) in the validity of our results, we can now proceed with the interpretation of Fig. 7 which shows the distribution of dissipation rate and vertical wind shear within and around the frontal zone. Some features in Fig. 7(a) to note are:

- The largest values of $\epsilon$, i.e. $\epsilon>3 \times 10^{-3} \mathrm{~m}^{2} \mathrm{~s}^{-3}\left(\log _{10} \epsilon>-2.5\right)$ are found in a layer close to the ground, the depth of which decreases from around 1.0 to $0.3 \mathrm{~km}$ shortly before the surface front passes over the radar at around 1000 UTC. This is at least qualitatively consistent with a mesoscale-model time-height section (shown later in Fig. 12), which shows a corresponding change across the front in the depth of the nearsurface layer containing low Richardson number values. It should be remembered that the model lags behind the observations by nearly one hour with regard to the location of the SCF.

- There is a $3 \mathrm{~km}$ deep region of enhanced dissipation rate, i.e. $8 \times 10^{-4}<\epsilon<$ $2 \times 10^{-3} \mathrm{~m}^{2} \mathrm{~s}^{-3}\left(-3.1<\log _{10} \epsilon<-2.7\right)$, whose top increases in height with time during and after the passage of the surface cold front; this broad region contains at least three distinct layers of enhanced dissipation rate (see dashed lines in Fig. 7(a)), and two more layers exist above this region.

- All of the layers of enhanced $\epsilon$ correspond to layers of increased vertical wind shear (Fig. 7(b)). Section 3(c) indicates that this is not due to contamination of $\sigma_{\mathrm{D}}$ by the shear, but is instead due to a real correspondence between shear layers and turbulence.

It is interesting to use these estimates of $\epsilon$ to quantify the effect of the turbulence on mean properties of the front, in particular the distribution of vertical shear. We do this by assuming that the turbulence acts to diffuse the velocity profile in a down-gradient sense.

Kennedy and Shapiro (1980) give the following expression for the turbulent kineticenergy budget, assuming that the vertical transport of energy $(E)$ by the turbulence itself is small:

$$
\frac{\partial\langle E\rangle}{\partial t}=-\langle v w\rangle \frac{\partial V}{\partial z}+\frac{g}{\theta_{0}}\left\langle w \theta^{\prime}\right\rangle-\epsilon
$$

where

$$
\langle v w\rangle=-K_{\mathrm{m}} \frac{\partial V}{\partial z} \quad \text { and } \quad\left\langle w \theta^{\prime}\right\rangle=-K_{\mathrm{h}} \frac{\partial \theta}{\partial z}
$$

define the eddy diffusion coefficients for momentum $\left(K_{\mathrm{m}}\right)$ and heat $\left(K_{\mathrm{h}}\right)$, respectively. The quantities $\theta_{0}$ and $\theta^{\prime}$ are the mean and perturbation potential temperatures; $V$ and $v$ are the mean and perturbation components of flow in the direction of the local shear vector. If we assume that the Prandtl number $\left(\operatorname{Pr}=K_{\mathrm{m}} / K_{\mathrm{h}}\right)$ is unity, and that the turbulent layer is in a steady state so that $\partial\langle E\rangle / \partial t=0$, then

$$
K=K_{\mathrm{m}}=\frac{\epsilon}{S^{2}(1-R i)^{-1}} \quad \text { with } \quad R i=\frac{g}{S^{2} \theta_{0}} \frac{\partial \theta}{\partial z}
$$

where $S$ is the magnitude of the vertical wind shear, and $R i$ is the Richardson number. Since we are principally interested in layers where turbulence exists, we assume that $R i=1 / 4$. Then we have a simple relationship between the eddy diffusivity, $K$, and the 
dissipation rate:

$$
K=\frac{4}{3} \frac{\epsilon}{S^{2}} .
$$

Using Fig. 9 we have $\epsilon=1.4 \times 10^{-3} \mathrm{~m}^{2} \mathrm{~s}^{-3}$ at a height of $2.6 \mathrm{~km}$, where the magnitude of the shear vector, $S$, is $19 \mathrm{~m} \mathrm{~s}^{-1} \mathrm{~km}^{-1}$. Therefore, we estimate the diffusion coefficient at this height to be $K \approx 5.2 \mathrm{~m}^{2} \mathrm{~s}^{-1}$.

We now assume that the effect of this turbulent diffusion on the component of shear parallel to the front (i.e. the thermal wind shear), $S_{\text {parr }}$, is given by

$$
\frac{\partial S_{\mathrm{par}}}{\partial t}=K \frac{\partial^{2} S_{\mathrm{par}}}{\partial z^{2}}
$$

where

$$
\frac{\partial^{2} S_{\mathrm{par}}}{\partial z^{2}} \approx-1 \times 10^{-7} \mathrm{~m}^{-2} \mathrm{~s}^{-1}
$$

has been estimated over the layer between 2 and $3 \mathrm{~km}$ using a centred difference approximation with the values of $S_{\mathrm{par}}$ taken from Fig. 9(a) at heights $2,2.5$ and $3 \mathrm{~km}$. In this way we estimate the rate of decrease in the shear in the centre of the frontal zone to be equal to around $2 \mathrm{~m} \mathrm{~s}^{-1} \mathrm{~km}^{-1}$ every hour. Thus, the time-scale for the tendency of turbulence to destroy this frontal shear layer is of order six hours, which is consistent with the high rate of frontogenesis which might be expected at an intense cold anafront such as this (e.g. Williams 1972), and with measurements within warm fronts (Browning et al. 1970).

It should be noted that the uncertainties involved in the derivation of this time-scale from $\epsilon$ are sufficiently large that the accuracy of this particular result should be treated with a considerable degree of caution. For example, each of the assumptions $R i=1 / 4$ and $\operatorname{Pr}=1$, as well as the estimate for $\partial^{2} S_{\text {par }} / \partial z^{2}$, could easily be in error by a factor of two, so even though we believe that our measurement of $\epsilon$ is quite accurate, the time-scale derived above is really an order-of-magnitude estimate. Thus it should be treated only as an indication that turbulence played a significant role in determining the properties of this frontal zone. If thermodynamic measurements could be obtained in addition to radar measurements of $\epsilon$, then a much more accurate estimation of the quantitative effects of the turbulence on the front could be made.

\section{CONCLUDING DISCUSSION}

(a) Measurements

This study demonstrates that it is possible to obtain quantitative estimates of dissipation rate using Doppler radar during the passage of precipitating frontal zones. Care has been taken to ensure that assumptions involved in the derivation of dissipation rate are reasonable, and this gives us some confidence in our conclusion that turbulence within the cold-frontal zone on 10 February 2000 played an important role in influencing the mean structure of the front.

Previous estimates of dissipation rate from Doppler spectrum widths measured with microwave radar have generally been made in thunderstorm environments, where the relatively large outer scale length permits the use of radars with an angular resolution considerably poorer than that of the Chilbolton radar. The cross-sections shown in this 


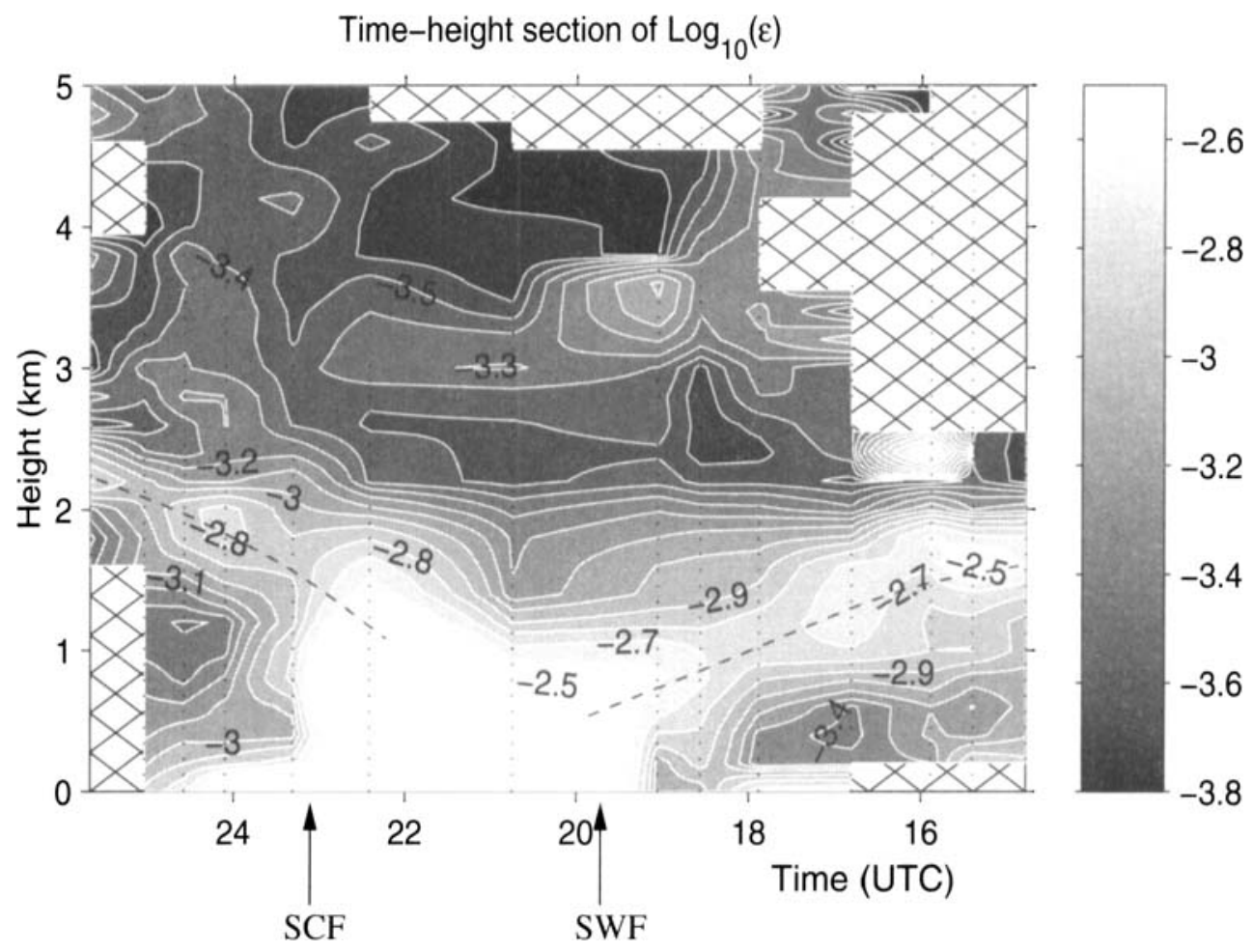

Figure 10. Time-height section over Chilbolton of the logarithm of the dissipation rate, $\epsilon,\left(\mathrm{m}^{2} \mathrm{~s}^{-3}\right)$ during the passage of a frontal system on 24 February 2000 . Vertical dotted lines show the times of the profiles. The dashed lines draw attention to the layers of maximum $\epsilon$ associated with the warm-frontal zone (1500 to 2000 UTC) and the cold-frontal zone (2300 to 0200 UTC 25 February 2000). Also shown are the surface cold front (SCF) and the surface warm front (SWF). The hatched regions show where there were insufficient data.

paper indicate that the mixing occurring within frontal shear zones can consist of many turbulent sub-layers or sheets, each only a few hundred metres or less thick. Thus, the inertial subrange within such regions will have an outer scale that will be similar to (or less than) this thickness. This would impose a very severe range constraint on the use of more conventional radars which have a beam width of, say, $1^{\circ}$, unless an accurate way can be found to estimate the outer scale length from the resolved properties of the flow.

For the purposes of measuring dissipation rates the main advantage that the Chilbolton radar has over other radars is its high spatial resolution. This means that we need only to be confident that the outer scale length is greater than a certain value in order to measure $\epsilon$, rather than needing an accurate estimate of the outer scale length. Therefore, in principle it is straightforward to make dissipation rate measurements through any precipitating frontal zone.

As a further example, we show in Fig. 10 a time-height section similar to that derived in our main study (Fig. 7) but through a developing frontal wave on 24 February 2000 . The location of the warm-frontal zone can be clearly seen over the radar between 1500 and 2000 UTC as a sloping zone of enhanced dissipation rate (dashed line), the magnitude of which is similar to that in the 10 February cold-frontal case (Fig. 7(a)). It is reassuring that during this period the dissipation rates near to the ground are rather low compared with the main case-study; if the large dissipation rates measured near the surface in the 10 February case had been due to Doppler spectrum 'contamination' by ground clutter, one might have expected a similar effect in this case, too. The surface 


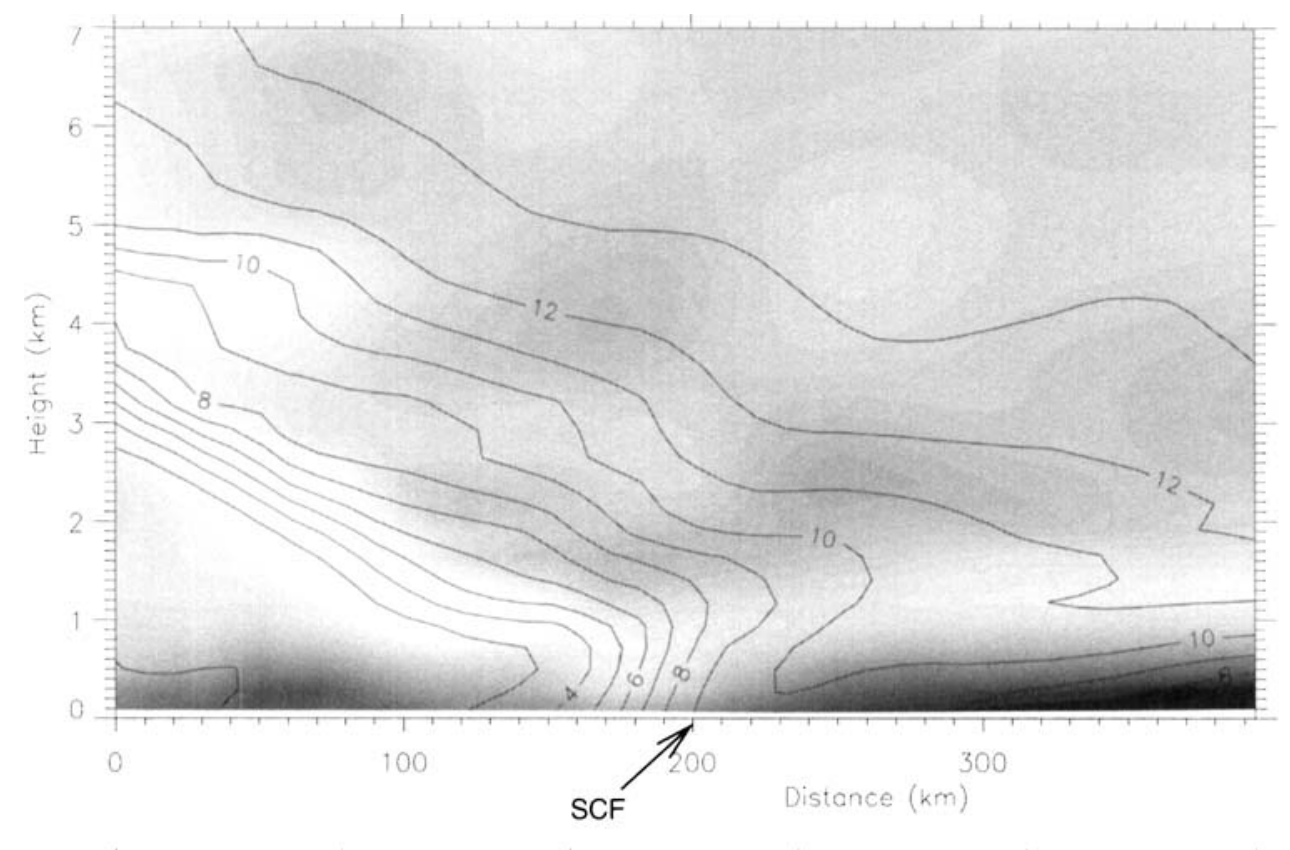

Figure 11. Cross-section through the six-hour mesoscale-model forecast of the cold front on 10 February 2000 , valid at 1100 UTC. Contours show wet-bulb potential temperature $\left({ }^{\circ} \mathrm{C}\right)$ and shading indicates the horizontal wind component parallel to this plane. The location of the model's surface cold front (SCF) is marked, and Chilbolton is situated near the centre of this section.

warm front on 24 February reached the radar around 2000 UTC, which coincided with the onset of a period of strong dissipation near the surface $\left(\epsilon \approx 10^{-2} \mathrm{~m}^{2} \mathrm{~s}^{-3}\right)$, after which the layer of strong dissipation associated with the cold-frontal zone can be seen rising to a height of around $2.2 \mathrm{~km}$ by the end of the observing period.

\section{(b) Implications for NWP model development}

It is interesting to compare the radar observations from the main case-study in this paper with output from the operational mesoscale version of the Met Office UM (Cullen 1993). This model is hydrostatic with a $12 \mathrm{~km}$ grid and 38 levels in the vertical, and is nested within the global version of the UM which has a $60 \mathrm{~km}$ grid in the midlatitudes. Figure 11 shows a vertical cross-section through the front in the mesoscale model, after interpolation onto constant-pressure surfaces which are spaced at intervals of $25 \mathrm{hPa}$. This cross-section passes through the Chilbolton radar, and is in the same direction as the radar cross-section in Fig. 3. A single thermally direct circulation dominates the model's representation of the transverse mesoscale frontal structure, with a well defined sloping shear layer centred just below $2 \mathrm{~km}$ about $100 \mathrm{~km}$ behind the SCF. Although there are clearly a number of perturbations to this structure on the warm-air side of the frontal zone, the strength of these perturbations is considerably weaker than in the radar observations, and they occur on single model levels rather than with a substantial slope as observed. 

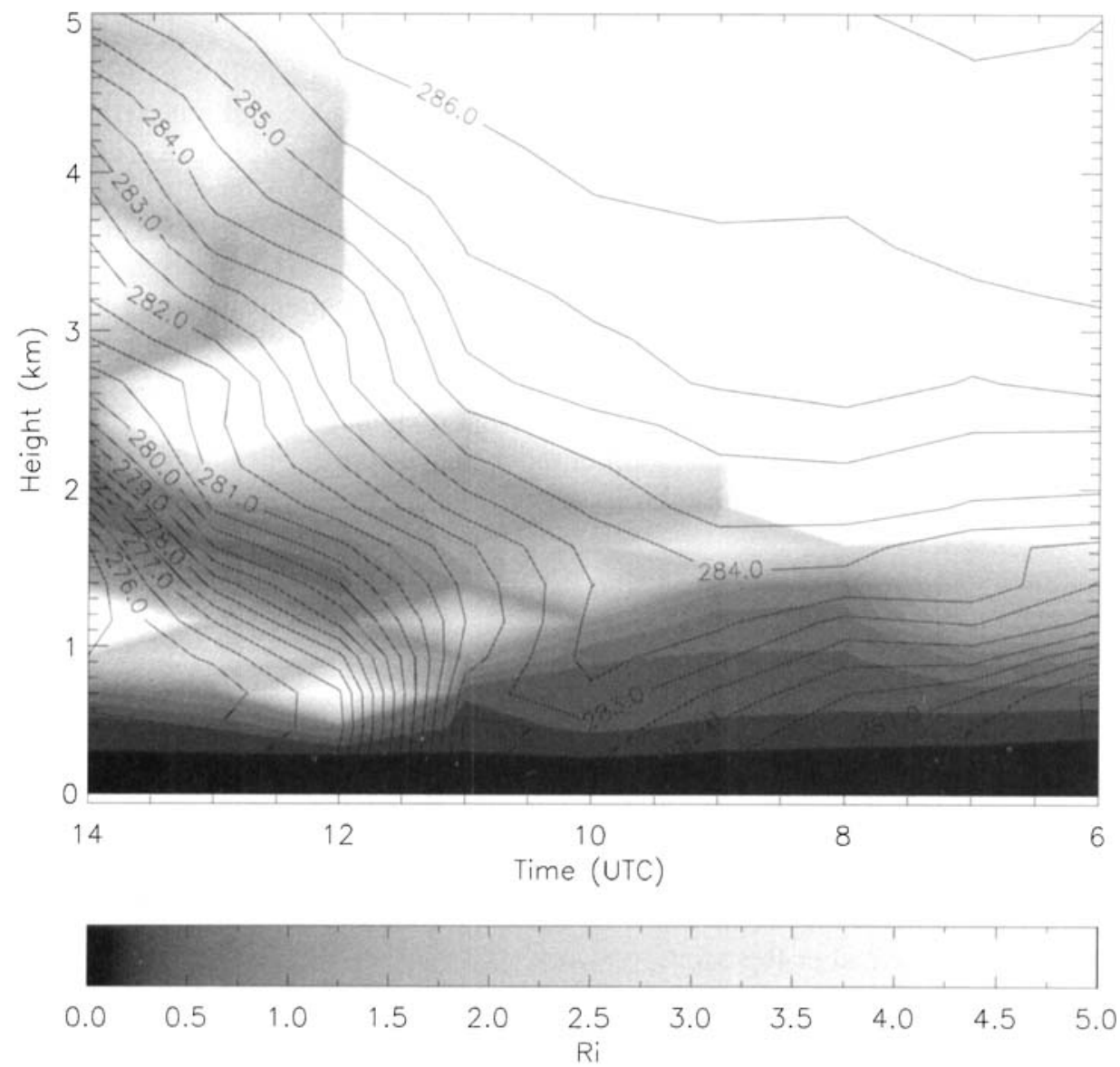

Figure 12. Time-height section over the Chilbolton radar of wet-bulb potential temperature (contoured at $0.5 \mathrm{~K}$ intervals) and Richardson number (shaded according to the key) from the 0600 UTC mesoscale-model forecast on 10 February 2000.

Figure 12 shows a time-height section over Chilbolton of wet-bulb potential temperature $\left(\theta_{\mathrm{w}}\right)$ and Richardson number $(R i)$ from the mesoscale model. When comparing this plot with the observations in Fig. 7, one can see that there is a marked decrease in the depth of the model boundary layer shortly before the SCF passes over the radar location (as noted in section 3(d)), and the sloping zone of low $R i$ values within the frontal zone corresponds to the broad region of enhanced dissipation in the radar time-height section. However, when viewed in this way, the model data do not show any evidence of multiple layers of low $R i$ that might correspond to the multiple layers seen in the dissipation rate sections: in fact it has a uniformly high $R i$ in the region above and ahead of the frontal zone, where the observations indicated significant mixing. This is to be expected, given that in Fig. 11 the model only weakly represents the multiple circulations that were very clear in the radar cross-sections. Thus it appears that attempting to predict regions of turbulence based on the mesoscale model's Richardson number will lead to poor results because of the model's inability to represent the mesoscale circulations that lead to the turbulent shear layers.

The amount of horizontal diffusion that is used in the current mesoscale model means that it is capable of representing features only down to scales of a few times the 
grid length. Recent trials with the so-called 'new-dynamics' version of the Met Office mesoscale model show that it is possible to run the model with no artificial diffusion and with a grid length of $4 \mathrm{~km}$ or less ( $\mathrm{H}$. Lean and P. Clark, personal communication). Lean and Clark show that the new model (a) appears to represent multiple frontal structures better and (b) can collapse fronts down to the grid scale. If the model can correctly represent the structures that are directly responsible for causing turbulent dissipation in the atmosphere, it should in principle be possible to infer dissipation rates directly from the model using a parametrization scheme. Thus, frontogenesis would be limited to a scale that is determined by the physical processes associated with the front, rather than the model parameters such as grid size. Validation of such a parametrization requires measurements of both dissipation rate and the dynamical background; we have demonstrated that a high-resolution Doppler radar such as the one at Chilbolton provides an excellent tool for obtaining these kinds of measurements.

\section{ACKNOWLEDGEMENTS}

We are very grateful to Andrew Openshaw and the Chilbolton radar staff for developing the radar hardware and software used to collect the data presented in this study. Peter Panagi's help in providing the Met Office mesoscale-model data and processing software is highly valued. We thank the Met Office for supporting this work under contract Agreement No. Met1b/2617. This is a development of work started under the Natural Environment Research Council (NERC) Grant GST/02/2215. The Joint Centre for Mesoscale Meteorology is supported by the Meteorological Office and the Department of Meteorology, University of Reading, and by NERC through its support of the Universities Weather Research Network.

\section{Blumen, W.}

Blumen, W. and Piper, M. Bohne, A. R.

Brewster, K. A. and Zrnić, D. S.

Browning, K. A.

Browning, K. A., Harrold, T. W. and Starr, J. R.

Browning, K. A., Roberts, N. M. and Illingworth, A. J.

Bryant, G. W. and Browning, K. A.

Chapman, D. and Browning, K. A.
1990

1986

1990

1970

1997

1975

1997

1998

1999

\section{REFERENCES}

A semigeostrophic Eady-wave frontal model incorporating momentum diffusion. Part II: Kinetic energy and enstrophy dissipation. J. Atmos. Sci., 47, 2903-2908

The frontal width problem. J. Atmos. Sci., 56, 3167-3172

'Estimation of turbulence severity in precipitation environments by radar'. Pp. 446-453 in Proceedings of the 20th Conference on Radar Meteorology, Am. Meteorol. Soc.

Radar detection of turbulence in precipitation environments. J. Atmos. Sci., 39, 1819-1837

Comparison of eddy dissipation rates from spatial spectra of Doppler velocities and Doppler spectrum widths. J. Atmos. Oceanic Tech., 3, 440-452

Chapter 8: Organisation of clouds and precipitation in extratropical cyclones. Pp. 129-153 in Extratropical cyclones. Eds. C. W. Newton and E. O. Holopainen, Am. Meteorol. Soc., Boston, Mass., USA

Richardson number limited shear zones in the free atmosphere. Q. J. R. Meteorol. Soc., 96, 40-49

Mesoscale analysis of the activation of a cold front during cyclogenesis. Q. J. R. Meteorol. Soc., 123, 2349-2375

Multi-level measurements of turbulence over the sea during the passage of a frontal zone. Q.J.R. Meteorol. Soc., 101, 3554

Radar observations of wind-shear splitting within evolving atmospheric Kelvin-Helmholtz billows. Q. J. R. Meteorol. Soc., 123, 1433-1439

Use of wind-shear display for Doppler radar data. Bull. Am. Meteorol. Soc., 79, 2685-2691

Release of potential shearing instability in warm frontal zones. Q. J. R. Meteorol. Soc., 125, 2265-2290 
Chapman, D., Roberts, N. M., Illingworth, A. J. and Browning, K. A.

Clarke, R. H.

Cullen, M. J. P.

Doviak, R. J. and Zrnić, D. S. Frisch, A. S. and Clifford, S. F.

Gage, K. S., Green, J. L. and VanZandt, T. E.

Goddard, J. W. F., Eastment, J. D. and Thurai, $\mathbf{M}$.

Gossard, E. E.

Keller, J. L.

Kennedy, P. J. and Shapiro, M. A.

Labitt, $\mathbf{M}$.

Lhermitte, R. M. and Atlas, D.

Meischner, P., Baumann, R., Höller, H. and Jank, T.

Pepler, S. J., Vaughan, G. and Hooper, D. A.

Roach, W. T.

Srivastava, R. C. and Atlas, D.

Srivastava, R. C., Jameson, A. R. and Hildebrand, P. H.

Williams, R. T.
1998 First radar detection of a misocyclone in a UK tomadic storm. Weather, 53, 367-368
1966

1993

1984

1974

1980

1994

1990

1989

1975

1980

2001

1998

1972
Turbulence and detailed structure of a subtropical jet stream. J. Atmos. Sci., 23, 516-530

The unified forecast/climate model. Meteorol. Mag., 122, 81-94

Doppler radar and weather observations. Academic Press

A study of convection capped by a stable boundary layer using Doppler radar and acoustic echo sounders. J. Atmos. Sci., 31, $1622-1628$

Use of a doppler radar for the measurements of atmospheric turbulence parameters from the intensity of clear-air echoes. Radio. Sci., 15, 407-416

The Chilbolton advanced meteorological radar: A tool for multidisciplinary research. Electronics and Communications Engineering Journal, 6, 77-86

Chapter 27a: Radar research on the atmospheric boundary layer. Pp. 477-527 in Radar in meteorology. Ed. D. Atlas, Am. Meteorol. Soc.

Clear air turbulence as a response to meso- and synoptic-scale dynamic processes. Mon. Weather Rev., 18, 2228-2242

The energy budget in a clear air turbulence zone as observed by aircraft. Mon. Weather Rev., 103, 650-654

Further encounters with clear air turbulence in research aircraft. J. Atmos. Sci., 37, 986-993

'Coordinated radar and aircraft observations of turbulence'. Project report ATC 108. Massachusetts Institute of Technology, Lincoln Lab., Cambridge, USA

'Precipitation motion by pulse Doppler'. Pp. 218-223 in Proceedings of the Ninth Weather Radar Conference, Am. Meteorol. Soc.

Eddy dissipation rates in thunderstorms estimated by Doppler radar in relation to aircraft in situ measurements. J. Atmos. Ocean. Tech., in press

Detection of turbulence around jet streams using a VHF radar. Q. J. R. Meteorol. Soc., 124, 447-462

On the influence of synoptic development on the production of high level turbulence. $Q$. J. R. Meteorol. Soc., 96, 413-429

Effect of finite radar pulse volume on turbulence measurements. J. Appl. Meteorol., 13, 472-480

Time-domain computation of mean and variance of Doppler spectra. J. Appl. Meteorol., 18, 189-194

Quasi-geostrophic versus non-geostrophic frontogenesis. J. Atmos. Sci., 29, 3-10 